\title{
Measurement of Temporal Correlations of the Overhauser Field in a Double Quantum Dot
}

\author{
D. J. Reilly ${ }^{1}$, J. M. Taylor ${ }^{2}$, E. A. Laird ${ }^{1}$, J. R. Petta ${ }^{3}$, C. M. Marcus ${ }^{1}$, M. P. Hanson ${ }^{4}$ and A. C. Gossard ${ }^{4}$ \\ 1 Department of Physics, Harvard University, Cambridge, MA 02138, USA \\ 2 Department of Physics, Massachusetts Institute of Technology, Cambridge, MA 02139, USA \\ 3 Department of Physics, Princeton University, Princeton, NJ 08544, USA and \\ 4 Department of Materials, University of California, Santa Barbara, California 93106, USA
}

\begin{abstract}
In quantum dots made from materials with nonzero nuclear spins, hyperfine coupling creates a fluctuating effective Zeeman field (Overhauser field) felt by electrons, which can be a dominant source of spin qubit decoherence. We characterize the spectral properties of the fluctuating Overhauser field in a GaAs double quantum dot by measuring correlation functions and power spectra of the rate of singlet-triplet mixing of two separated electrons. Away from zero field, spectral weight is concentrated below $10 \mathrm{~Hz}$, with $\sim 1 / f^{2}$ dependence on frequency, $f$. This is consistent with a model of nuclear spin diffusion, and indicates that decoherence can be largely suppressed by echo techniques.
\end{abstract}

Electron spins in quantum dots are an attractive candidate for quantum bits (qubits) [1, 2]. For gate-defined devices made using GaAs, the coupling of single electron spins to $\sim 10^{6}$ thermally excited nuclear spins creates a fluctuating effective Zeeman field (the Overhauser field), $\mathbf{B}_{n u c}$, with rms amplitude $B_{n u c} \sim 1-3 \mathrm{mT}[3,4,5,6,6]$. At experimentally accessible temperatures, $\mathbf{B}_{n u c}$ fluctuates both as a function of position and time, with temporal correlations over a broad range of time scales, and is a dominant source of spin dephasing [8, 9, 10, 11, 12, 13. and low-field spin relaxation [3, 4, 7, 14, 15, 16] in these systems. Spin manipulation schemes [10, 17, 18, 19] to control spin dephasing, such as spin echo and its generalizations, depend critically on a knowledge of correlations and time scales of the fluctuating nuclear environment.

Previously, fluctuating Overhauser fields have been investigated in atomic systems [20] using optical Faraday rotation, superconducting quantum interference devices 21] and force-detected magnetic resonance 22]. In quantum dots, dynamic nuclear polarization (DNP) 23, 24, 25, 26] can drive the nuclear system beyond equilibrium to produce fluctuating currents and feedback effects in connection with Pauli spin-blockade [15, 27, 28, 29.

In this Letter, we report measurements of the temporal correlations and power spectral densities of the nuclear environment in a two-electron GaAs double-quantum-dot system. In contrast to previous work [15, 25, 26], we do not drive the nuclear system using DNP, but rather probe the statistical fluctuations of the unpolarized nuclear bath in thermal equilibrium [8, 12. Fluctuations of the Overhauser field are detected as fluctuations in the dephasing time of a two-electron spin state, making use of high-bandwidth proximal charge sensing [30. Fluctuations are found to be broadband over the measurement bandwidth, $40 \mathrm{mHz}$ to $1 \mathrm{kHz}$, and sensitive to an applied magnetic field in the range $B=0$ to $20 \mathrm{mT}$. Experimental results are shown to be consistent with a simple diffusion model of nuclear dynamics, also presented here.

The double quantum dot is formed by $\mathrm{Ti} / \mathrm{Au}$ top gates on a $\mathrm{GaAs} / \mathrm{Al}_{0.3} \mathrm{Ga}_{0.7} \mathrm{As}$ heterostructure with a two-dimensional electron gas (2DEG) with density $2 \times 10^{15} \mathrm{~m}^{-2}$ and mobility $20 \mathrm{~m}^{2} / \mathrm{Vs}, 100 \mathrm{~nm}$ below the surface (Fig. 1(a), inset), similar to devices reported previously [9, 26. Measurements are made in a dilution refrigerator with base electron temperature of $\sim 120 \mathrm{mK}$. The conductance $G_{\mathrm{QPC}}$ of a proximal radio frequency quantum point contact (rf-QPC) is sensitive to the charge configuration of the double dot. $G_{\mathrm{QPC}}$ controls the quality factor of an rf tank circuit, modulating the reflected power of a $220 \mathrm{MHz}$ carrier. Demodulation yields a voltage $V_{\mathrm{rf}}$, proportional to $G_{\mathrm{QPC}}$, that constitutes the charge-sensing signal [30]. The applied field, $B$, is oriented perpendicular to the 2DEG.

Figure 1(a) shows the relevant energy levels of the double dot in the vicinity of the $(2,0)-(1,1)$ charge transition [first (second) index is the charge in the left (right) dot]. Interdot tunneling, $t_{c}$, and detuning, $\epsilon$, from the charge degeneracy are determined by electrostatic gates. A gate-pulse (Fig. 1(b)) cycle prepares new singlets each iteration by configuring the device deep in $(2,0)$, at point $(\mathrm{P})$, where transitions to the ground state singlet, $(2,0) S$, occur rapidly [14. Electrons are then separated to position $\mathrm{S}$ in $(1,1)$ for a time $\tau_{\mathrm{S}}$ where precession between the initial singlet and one of the triplet states is driven by components of the difference in Overhauser fields in the left and right dots, $\Delta \mathbf{B}_{n u c}=\mathbf{B}_{n u c}^{l}-\mathbf{B}_{n u c}^{r}$ [9, 13].

In an applied field, the position of the separation point determines whether the $(1,1)$ singlet $(S)$ is nearly degenerate with one of the $(1,1)$ triplets, with which it can then rapidly mix. Mixing of $S$ with $T_{0}$ (the $m_{s}=0$ triplet) occurs at large negative $\epsilon$ (green line in Fig. 1(b)) where exchange vanishes. $S-T_{0}$ mixing is driven by components of $\Delta \mathbf{B}_{n u c}$ along the total field (applied plus Overhauser fields). In contrast, mixing of $S$ with $T_{+}$(the $m_{s}=+1$ triplet), which occurs at a less negative, fielddependent value of $\epsilon$ (red line in Fig. 1(b)) where Zeeman splitting matches exchange, is driven by components of $\Delta \mathbf{B}_{\text {nuc }}$ transverse to the total field. Measuring the degree of evolution out of the prepared $S$ state following separation, by measuring the return probability to the $(2,0)$ 


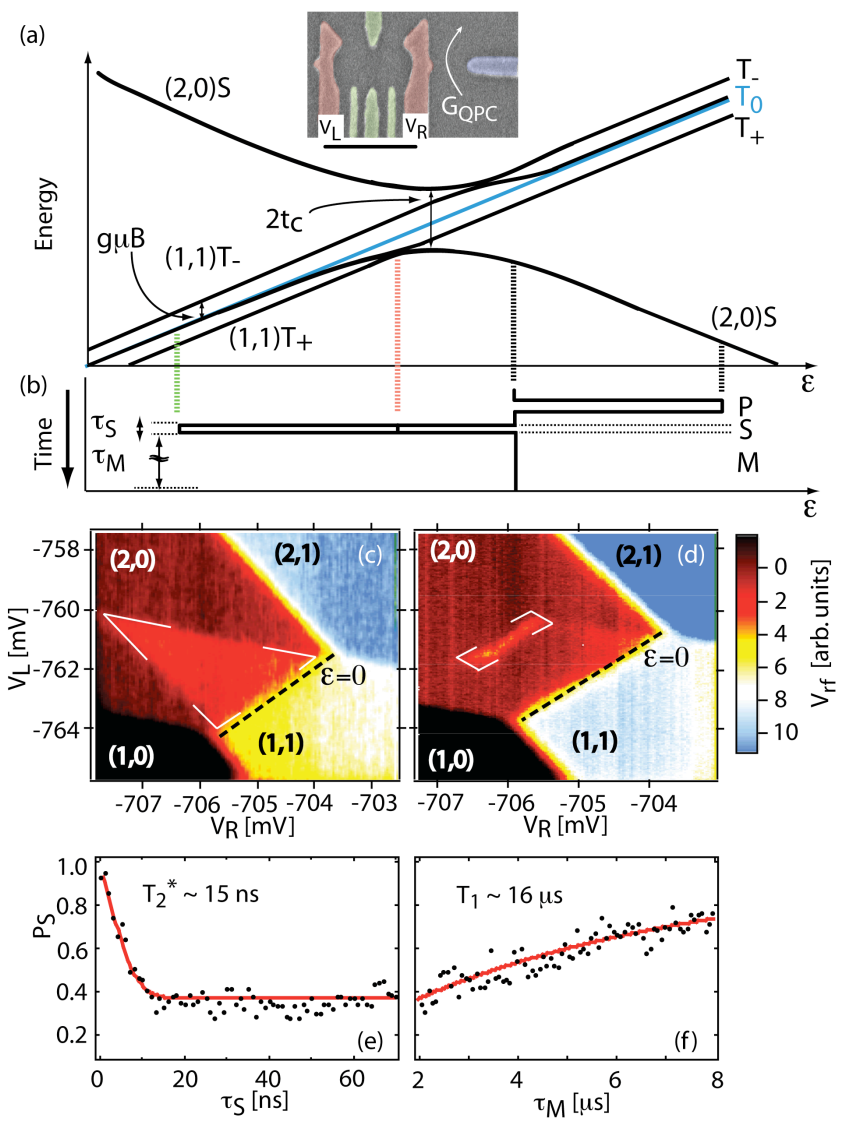

FIG. 1: (Color online) (a) Schematic energy diagram of the two-electron system. Inset: false-color SEM image of a double-dot with integrated rf-QPC charge sensor similar to the one measured (scale bar is $500 \mathrm{~nm}$ ). (b) Gate-pulse cycle that is used to prepare $(\mathrm{P})$ the $(2,0)$ singlet, separate $(\mathrm{S})$ into $(1,1)$, either to the $S-T_{0}$ degeneracy (green dashed line) or the $S-T_{+}$degeneracy (red dashed line), and return to $(2,0)$ for measurement (M). (c) rf-QPC readout, $V_{\mathrm{rf}}$, around the $(1,1)-(2,0)$ transition during application of the cyclic gatepulse sequence, showing the readout triangle indicated with white lines $\left(B=0 \mathrm{mT}, \tau_{\mathrm{S}}=50 \mathrm{~ns}\right)$. A background plane has been subtracted. (d) $V_{\text {rf }}$ as in (c), but for S at the $S-T_{+}$ degeneracy $(B=10 \mathrm{mT})$. (e) Average value of $P_{S}\left(\tau_{\mathrm{S}}\right)$ at $B=$ $0, \tau_{\mathrm{M}}=2 \mu \mathrm{s}$. Red line is a fit to the theoretical gaussian form. (f) Average value of $P_{S}\left(\tau_{\mathrm{M}}\right)$ showing contrast dependence, $\tau_{\mathrm{S}}$ $=50 \mathrm{~ns}$. Red line is a fit to the exponential form (see main text).

charge configuration after a certain separation time, effectively measures these components of the Overhauser field difference in the two dots. Measurement is carried out by moving the system to position $\mathrm{M}$ in $(2,0)$ for a time $\tau_{\mathrm{M}}=5 \mu \mathrm{s}$, during which only $S$ return to $(2,0)$ with appreciable probability. The spin state - triplet or singlet - is thereby converted to a charge state- $(1,1)$ or $(2,0)$, respectively - which is detected by the rf-QPC.

Figures $1(\mathrm{c}, \mathrm{d})$ show the time-averaged $V_{\text {rf }}$ as a function of gate voltages $V_{L}$ and $V_{R}$. Once calibrated, $V_{\text {rf }}$ gives the probability $1-P_{S}$ that a prepared singlet evolved into a triplet during the separation time $\tau_{\mathrm{S}}$. In-
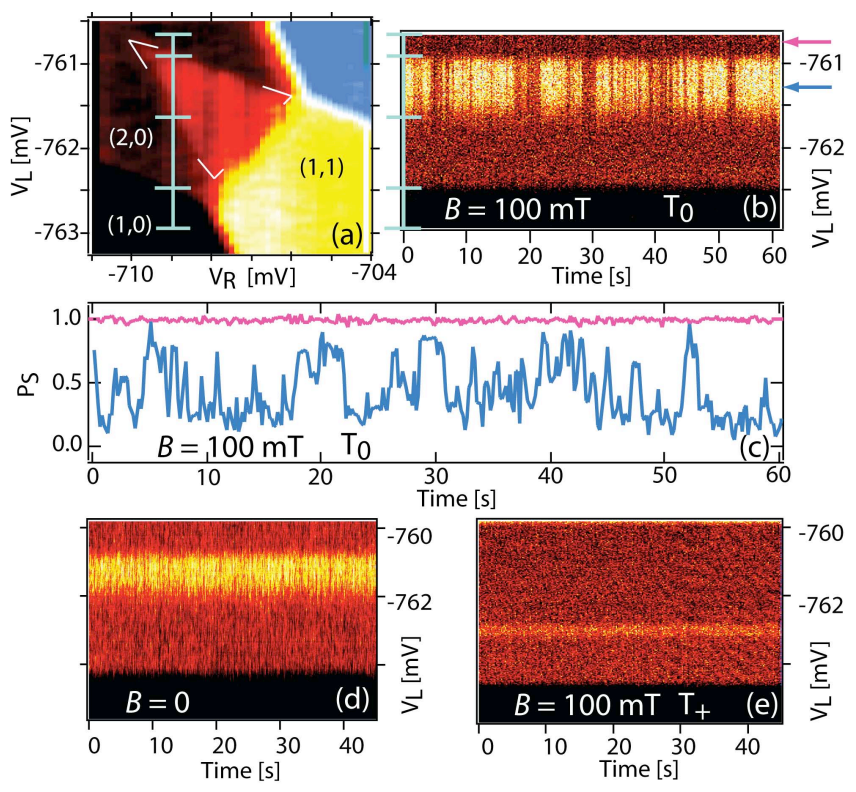

FIG. 2: (Color online) (a) rf-QPC sensor output $V_{\text {rf }}$ as a function of $V_{L}$ and $V_{R}$ with gate-pulse cycle applied $\left(\tau_{\mathrm{S}}=25 \mathrm{~ns}\right.$, $\tau_{\mathrm{M}}=1.6 \mu \mathrm{s}, B=100 \mathrm{mT}$ ). Color scale as in Fig. 1. (b) Repeated slices of $V_{L}$ with $V_{R}=-709 \mathrm{mV}$ as a function of time. Markers on left axis correspond to markers in (a). (c) Sensor output calibrated to $P_{S}$ (blue) along with a measurement of the background QPC noise (pink) from (b) at arrow positions. Bandwidth limited to $\sim 3 \mathrm{~Hz}$. (d) Similar to (b) but for $B=0$, color scale same as in Fig. 1. (e) Similar to (b) but with S-point at $S-T_{+}$degeneracy, $B=100 \mathrm{mT}$, color scale same as in Fig. 1.

side the readout triangle (see Fig. 1(c)), triplet states remain blocked in $(1,1)$ for a time $T_{1} \gg \tau_{\mathrm{M}}$ [14. Similarly, inside the rectangular region indicated in Fig. 1(d), the prepared singlet mixes with $T_{+}$and becomes blocked in $(1,1)$. Calibration of $V_{\mathrm{rf}}$ uses the signal in $(2,0)$ outside the readout triangle, where fast, spin-independent relaxation occurs via $(1,0)$ or $(2,1)$, to define $P_{S}=1$, and the region within $(1,1)$ to define $P_{S}=0$.

Fitting $P_{S}\left(\tau_{\mathrm{S}}\right)$ averaged over tens of seconds with a gaussian [9, 13] (Fig. 1(e)) gives $T_{2}^{*}=$ $\hbar /\left(g \mu_{B} B_{\text {nuc }}\right) \sim 15 \mathrm{~ns}$ corresponding to $B_{\text {nuc }} \sim 1.6 \mathrm{mT}$ $\left(N \sim 6 \times 10^{6}\right)$, where $g \sim-0.4$ is the electron $g$-factor and $\mu_{B}$ is the Bohr magneton. The effect of finite $T_{1}$ on the calibration of $P_{S}$ can be accounted by introducing a factor $C=\left(1-e^{-\tau_{\mathrm{M}} / T_{1}}\right) T_{1} / \tau_{\mathrm{M}}$ 14 that relates $P_{S}$ to the value $P_{S}^{\prime}$ corresponding to infinite $T_{1}, 1-P_{S}=\left(1-P_{S}^{\prime}\right) C$. The dependence of $P_{\mathrm{S}}$ on $\tau_{\mathrm{M}}$ (for a fixed $T_{1} \sim 16 \mu \mathrm{s}$ and $\left.\tau_{\mathrm{S}}=50 \mathrm{~ns}\right)$ is shown in Fig. 1(f). Applying this factor to Fig. 1(e) gives $P_{S}^{\prime}\left(\tau_{\mathrm{S}} \gg T_{2}^{*}\right)=1 / 3$, the expected value [13], without normalizing the sensor output.

With less averaging, $P_{S}$ shows fluctuations that reflect fluctuations of Overhauser field components. Figure 2 shows a slice through the readout triangle, obtained by rastering $V_{L}$ at fixed $V_{R}$, with $B=100 \mathrm{mT}, \tau_{\mathrm{S}}=25 \mathrm{~ns}$. At $B=100 \mathrm{mT}$, fluctuations in $P_{S}$ have a flickering appearance with broadband time dependence extending to 

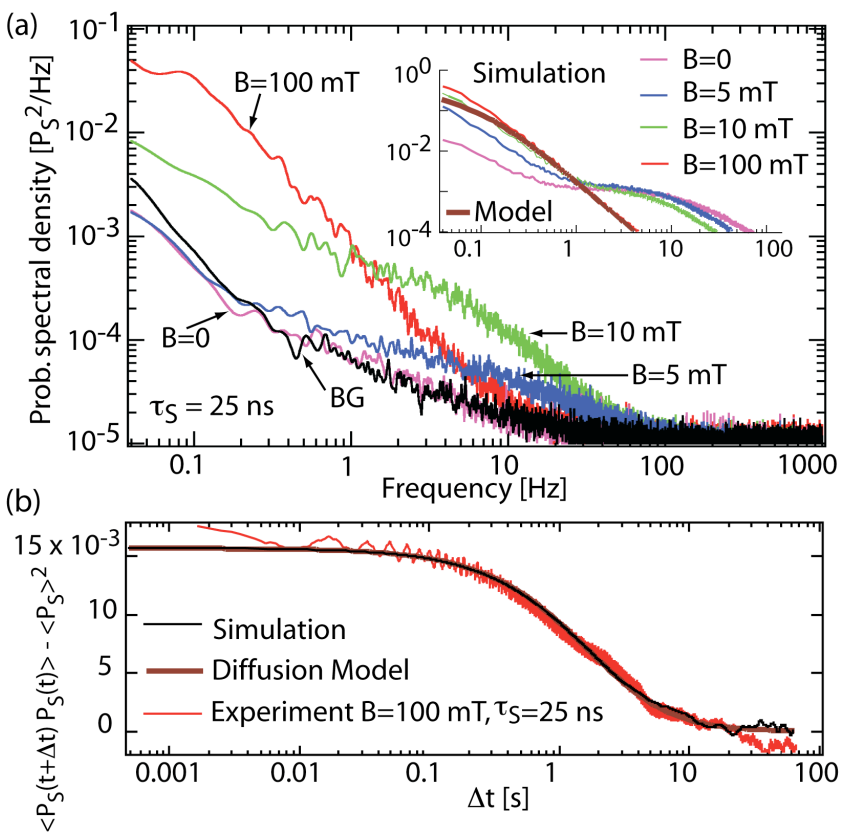

FIG. 3: (Color online) (a) Power spectra of $P_{S}$ at various magnetic fields, $\tau_{\mathrm{S}}=25$ ns. Spectra obtained by FFT (with Hamming window) of average of 8 traces sampled at $10 \mathrm{kHz}$. Background measurement noise (BG) found by setting $\tau_{\mathrm{S}}=1$ ns at $B=100 \mathrm{mT}$. Inset: numerical simulation results for corresponding magnetic fields: $B=0$ (pink), $B=5 \mathrm{mT}$ (blue), $B=10 \mathrm{mT}$ (green), $B=100 \mathrm{mT}$ (red). (b) Autocorrelation $P_{S}$ for $\tau_{\mathrm{S}}=25 \mathrm{~ns}$ and $B=100 \mathrm{mT}$ (red curve). Model function (Eq. 1) (brown) and Monte Carlo result (black).

several seconds. Comparing the quieter (pink) trace in Fig. 2c, for point $\mathrm{M}$ such that $(1,1)$ always returns to $(0,2)$, to the fluctuating (blue) trace, where return to $(0,2)$ requires $S-T_{0}$ mixing by Overhauser fields, we see that the amplitude of the fluctuating signal (blue) is $\sim 100$ times larger than the background noise of the charge sensor. At $B=0$, slices across the readout triangle does not show a flickering (large, low-frequency) $P_{S}$ signal (Fig. 2(d)). Figure 2(e) shows slices across the $S-T_{+}$resonance (see Fig. 1(d)). Here also, $P_{S}$ also does not have a flickering appearance, independent of $B$, reflecting rapid fluctuations of transverse components of $\Delta \mathbf{B}_{n u c}$. We avoid rapidly cycling through the $S-T_{+}$ transition, which can produce DNP [26.

To investigate the spectral content of $P_{S}$ fluctuations, fast Fourier transforms (FFTs) of $V_{\text {rf }}$ are taken with $V_{L}$ and $V_{R}$ positioned to sample the center of the readout triangle. Figure 3(a) shows power spectra of $P_{S}$, with $\tau_{\mathrm{S}}=$ $25 \mathrm{~ns}$, over the range $B=0-100 \mathrm{mT}$. Measurement at $\tau_{\mathrm{S}}$ $=1 \mathrm{~ns}$, where $P_{S} \sim 1$, has a $1 / f$ form and is identical to the noise measured outside the readout triangle, and constitutes our background of instrumental noise. At $B=0$ no spectral content above the $1 / f$ background noise is seen (Fig. 2(a)). With increasing $B$, an increasing spectral content is observed below $\sim 100 \mathrm{~Hz}$. For $B>20$ $\mathrm{mT}$, the spectra become independent of $B$. The dependence of the power spectrum of $P_{S}$ on separation time $\tau_{\mathrm{S}}$ is shown in Fig. 4. We found that the largest fluctuations over the greatest frequency range occur for $\tau_{\mathrm{S}} \sim T_{2}^{*} \sim$ $15 \mathrm{~ns}$, and these fluctuations show a roughly $1 / f^{2}$ spectrum. Spectra were also obtained out to $100 \mathrm{kHz}$ (not shown) where no additional high frequency components were observed above the background noise. For $\tau_{\mathrm{S}}<T_{2}^{*}$, $P_{S}$ remains near unity with few fluctuations; For $\tau_{\mathrm{S}}>T_{2}^{*}$ low-frequency content is suppressed while components in the range $1-10 \mathrm{~Hz}$ are enhanced.

We model fluctuations in $P_{S}$ as arising from the dynamic Overhauser magnetic field in thermal equilibrium. A classical Langevin equation is used to describe fluctuations of $\Delta \mathbf{B}_{n u c}$ arising from nuclear spin diffusion on distances much larger than the lattice spacing and times much longer than the time-scale set by nuclear dipoledipole interaction. For $B \gg B_{n u c}$, correlations of the Overhauser field can be evaluated analytically in terms of a dimensionless operator $\hat{A}_{z}^{\beta}$ for each nuclear spin species $\beta$, where $\sum_{\beta} x^{\beta} \hat{A}_{z, l}^{\beta} \equiv \mathbf{B}_{n u c, z}^{l} / B_{n u c}$ and similarly for the right dot, with $x^{75} \mathrm{As}=1, x^{69} \mathrm{Ga}=0.6, x^{71} \mathrm{As}=0.4$. This gives $\left\langle\hat{A}_{z}^{\beta}(t+\Delta t) \hat{A}_{z}^{\beta}(t)\right\rangle=\left[\left(1+\Delta t D_{\beta} / \sigma_{z}^{2}\right)^{1 / 2}(1+\right.$ $\left.\left.\Delta t D_{\beta} / \sigma_{\perp}^{2}\right)\right]^{-1}$, at time difference $\Delta t$, where $D_{\beta}$ is the species-dependent spin diffusion coefficient, $\sigma_{z}$ is the electron wave function spatial extent perpendicular to the 2DEG (and along the external field) and $\sigma_{\perp}$ is the wave function extent in the plane of the $2 \mathrm{DEG}$, assumed symmetric in the plane. Brackets $\langle\ldots\rangle$ denote averaging over $t$ and nuclear ensembles.

Statistics of $P_{S}$ for $S-T_{0}$ mixing are found using the $z$-component of the Overhauser operators, $\Delta \hat{A}_{z}=$ $\sum_{\beta} x^{\beta}\left(\hat{A}_{z, l}^{\beta}-\hat{A}_{z, r}^{\beta}\right)$. For gaussian fluctuations and a species-independent diffusion constant, $D$, this gives a mean $\left\langle P_{S}\right\rangle=\frac{1}{2}\left[1+e^{-2 G^{2}\left\langle\Delta \hat{A}_{z}^{2}\right\rangle}\right]$ and autocorrelation $\left\langle P_{S}(t+\Delta t) P_{S}(t)\right\rangle-\left\langle P_{S}\right\rangle^{2}$

$$
=\frac{e^{-4 G^{2}\left\langle\Delta \hat{A}_{z}^{2}\right\rangle}}{4}\left[\cosh \left(4 G^{2}\left\langle\Delta \hat{A}_{z}(t+\Delta t) \Delta \hat{A}_{z}(t)\right\rangle\right)-1\right],
$$

where $G=\tau_{S} / T_{2}^{*}$ is a gain coefficient. The autocorrelation function at $B=100 \mathrm{mT}$ shown in Fig. 3(b) is obtained by Fourier transforming the power spectrum [31. We fit to the autocorrelation function using a contrast factor, $C$, (see Fig. 1(f) and discussion), and the diffusion coefficient, $D$, as fitting parameters. Wavefunction widths are taken from numerical simulations of the device [32], $\sigma_{z}=7.5 \mathrm{~nm}, \sigma_{\perp}=40 \mathrm{~nm}$. The fit gives $D \sim 10^{-13} \mathrm{~cm}^{2} / \mathrm{s}$, consistent with previous measurements on bulk GaAs samples using optical techniques [6]. In Eq. (1) the dependence on $\tau_{S}$ leads to a scaling of the correlation time of $P_{S}$ by $G^{2}$ to find the underlying Overhauser field correlation time. For fields $B>20 \mathrm{mT}$, the data in Fig. 3(b) indicate an autocorrelation time of $\sim 3 \mathrm{~s}$ for $P_{S}$ corresponding to a time $\tau_{d} \sim 10 \mathrm{~s}$ for $\Delta A_{z}$ to decorrelate by half of its initial value.

Near $B \sim 0$, transverse components of the nuclear field lead to rapid dephasing of nuclear spins. In this regime, we use a Monte Carlo method to simulate nuclear dynamics 33 . Figure 3(b) shows that numerical and analytical 


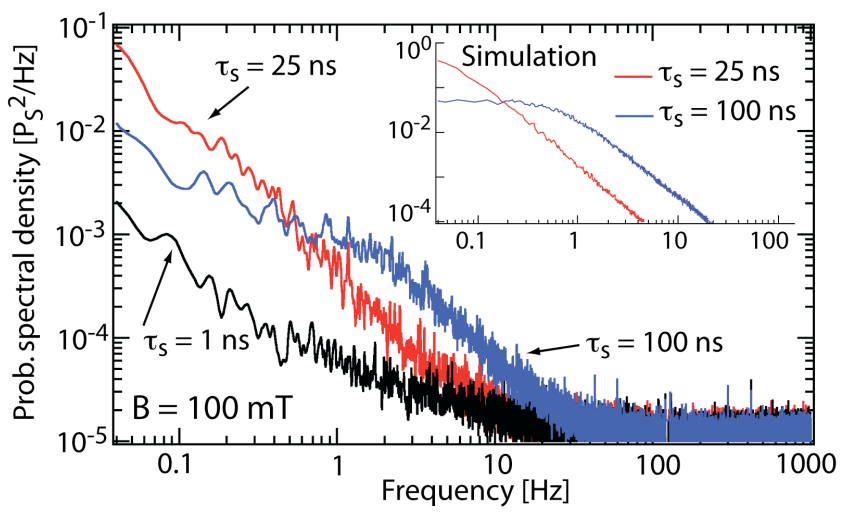

FIG. 4: (Color online) Power spectra of $P_{S}$ at $B=100 \mathrm{mT}$ for separation times $\tau_{\mathrm{S}}=25 \mathrm{~ns}$ (red) and $\tau_{\mathrm{S}}=100 \mathrm{~ns}$ (blue). Setting $\tau_{\mathrm{S}}=1 \mathrm{~ns}$ (black) yields background noise. Inset shows simulation results for $B=100 \mathrm{mT}, \tau_{\mathrm{S}}=25 \mathrm{~ns}(\mathrm{red})$ and $\tau_{\mathrm{S}}=$ $100 \mathrm{~ns}$ (blue). Note the suppression of low-frequency content and enhancement of mid-frequency content for long $\tau_{\mathrm{S}}$ in the experiment and simulation.

approaches agree at higher fields, where both methods are applicable. Numerical power spectra for $B \sim 0$ are shown in the inset of Fig. 4.

Experiment and theory show reduced low-frequency spectral content as $B$ decreases toward zero. This can be understood as arising from the influence of the transverse nuclear fields at low $B$, which rapidly dephase nuclear spins and suppress long time correlations in $\Delta B_{n u c}$. Similar behavior, though independent of $B$, is observed in the spectra of $P_{S}$ at the $S-T_{+}$resonance (not shown). Below $B \sim 10 \mathrm{mT}$, an increased spectral content at fre- quencies between $1-10 \mathrm{~Hz}$ is observed in the experiment and theory. The frequency at which the spectra intersect however, remains constant $(\sim 1 \mathrm{~Hz})$ in the simulations but increases at low $B$ in the experimental data. We are able to approximate this behavior in the simulation by increasing the diffusion coefficient $\left(D \sim 10^{-12} \mathrm{~cm}^{2} / \mathrm{s}\right.$ at $B=0$ ), implying an enhancement of diffusion, beyond typical values [6], as $B$ approaches zero. This may be due to the growing influence of non-secular terms in the dipole-dipole interaction at low magnetic field [8, 23]. Diffusion maybe further enhanced at low $B$ as a result of electron mediated flip-flop of nuclear spins [12, 34], an effect neglected in the simulation.

Finally, we model how the separation time for the twoelectron spin state affects the power spectra. Simulated spectra are shown in the inset of Fig. 4 for $\tau_{\mathrm{S}}=1 \mathrm{~ns}$, $25 \mathrm{~ns}$ and $100 \mathrm{~ns}$ at $B=100 \mathrm{mT}$. Good agreement with experiment is achieved when again accounting for the additional $1 / f$ noise and contrast reduction. We find that $\tau_{\mathrm{S}}$ acts to filter fluctuations in $\Delta B_{n u c}$, so that for $\tau_{S} \gg$ $T_{2}^{*}$, low frequency correlations in $\Delta B_{n u c}$ are suppressed in the spectra of $P_{S}$ (see Eq. 1). This filtering effect leads to the turn-over at $\sim 2 \mathrm{~Hz}$ evident in the spectra for $\tau_{\mathrm{S}}=$ $100 \mathrm{~ns}$. For $\tau_{S} \sim T_{2}^{*}$, little filtering occurs and the power spectra of $P_{S}$ reflect the underlying intrinsic fluctuations of the Overhauser magnetic field.

We thank L. DiCarlo, A. C. Johnson, and M. Stopa for contributions. This work was supported by DARPA, ARO/IARPA, NSF-NIRT (EIA-0210736) and Harvard Center for Nanoscale Systems. Research at UCSB supported in part by QuEST, an NSF Center.
[1] D. Loss and D. DiVincenzo, Phys. Rev. A. 57, 120 (1998).

[2] B. E. Kane, Nature (London) 393, 133 (1998).

[3] S. I. Erlingsson, Y. V. Nazarov, and V. I. Fal'ko, Phys. Rev. B. 64, 195306 (2001).

[4] I. A. Merkulov, A. L. Efros, and M. Rosen, Phys. Rev. B. 65, 205309 (2002).

[5] A. V. Khaetskii, D. Loss, and L. Glazman, Phys. Rev. Lett. 88, 186802 (2002).

[6] D. Paget, G. Lampel, and B. Sapoval, Phys. Rev. B. 15, 5780 (1977).

[7] A. S. Bracker et al., Phys. Rev. Lett. 94, 047402 (2005).

[8] R. de Sousa and S. Das Sarma, Phys. Rev. B. 68, 115322 (2003).

[9] J. R. Petta et al., Science 309, 2180 (2005).

[10] W. M. Witzel and S. Das Sarma, Phys. Rev. B. 74, 035322 (2006).

[11] F. H. L. Koppens et al., Nature (London) 442, 766 (2006).

[12] W. Yao, R.-B. Liu, and L. J. Sham, Phys. Rev. B. 74, 195301 (2006).

[13] J. M. Taylor et al., Phys. Rev. B. 76, 035315 (2007).

[14] A. C. Johnson et al., Nature (London) 435, 925 (2005).

[15] F. H. L. Koppens et al., Science 309, 1346 (2005).

[16] A. K. Huttel et al., Phys. Rev. B. 69, 073302 (2004).

[17] J. M. Taylor et al., Nature Physics (London) 1, 177
(2005).

[18] G. Giedke et al., Phys. Rev. A. 74, 032316 (2006).

[19] D. Klauser, W. A. Coish, and D. Loss, Phys. Rev. B. 73, 205302 (2006).

[20] S. A. Crooker et al., Nature (London) 431, 49 (2004).

[21] T. Sleator et al., Phys. Rev. Lett. 55, 1742 (1985).

[22] C. L. Degen et al., Phys. Rev. Lett. 99, 250601 (2007).

[23] A. Abragam, Principles of Nuclear Magnetism (International Series of Monographs on Physics) Oxford University Press, USA (1983).

[24] D. Gammon et al., Science 277, 85 (1997).

[25] J. Baugh et al., Phys. Rev. Lett. 99, 096804 (2007).

[26] J. R. Petta et al., Phys. Rev. Lett. 100, 067601 (2008).

[27] K. Ono and S. Tarucha, Phys. Rev. Lett. 92, 256803 (2004).

[28] M. S. Rudner and L. S. Levitov, Phys. Rev. Lett. 99, 036602 (2007).

[29] O. N. Jouravlev and Y. Nazarov, Phys. Rev. Lett. 96, 176804 (2006).

[30] D. J. Reilly et al., App. Phys. Lett. 91, 162101 (2007).

[31] The correlation function for the experimental data becomes slightly negative at long times, likely as the result of $1 / f$ noise in the rf-QPC.

[32] M. Stopa, Private communication, (2007).

[33] J. M. Taylor, unpublished. 
[34] C. Deng and X. Hu, Phys. Rev. B. 73, 241303(R) (2006). 